\title{
MANEJO AGRÍCOLA NO REFÚGIO DE VIDA SILVESTRE DE UNA: AGROFLORESTAS COMO UMA PERSPECTIVA DE CONSERVAÇÃO ${ }^{1}$
}

\author{
Isabel Sollberg², Alexandre Schiavetti ${ }^{3}$ e Maria Eugenia Bruck Moraes ${ }^{3}$
}

\begin{abstract}
RESUMO - Este estudo investiga os tipos de manejo e o perfil socioeconômico relacionado a estes, nas propriedades rurais do Refúgio de Vida Silvestre de Una. Tem-se como pressuposto de que as agroflorestas sucessionais são as mais favoráveis para conservação do bioma Mata Atlântica, inclusive na escala de paisagem. Portanto, objetiva-se conhecer os aspectos socioeconômicos associados à adoção de um tipo de manejo e sua inserção na paisagem. Para tanto, foram realizadas entrevistas em 73 propriedades, entre janeiro e maio de 2011. Os dados sobre os agrossistemas foram classificados em tipos de manejo: convencional, tradicional, orgânico e agroflorestal. Os resultados apontaram a predominância do tipo de manejo tradicional entre as propriedades. O perfil socioeconômico encontrado é compatível com as características do tipo de manejo predominante, o tradicional. Observaram-se no manejo tradicional: maior contribuição da mão de obra familiar e menor ocorrência de fonte de renda proveniente de trabalho rural para terceiros. A capacitação para atividade agrícola foi a única característica socioeconômica que apresentou maior importância para o manejo agroflorestal. Na paisagem, os resultados apontaram para a necessidade de adequação das práticas utilizadas nos agroecossistemas tradicionais aos objetivos da conservação do bioma, pois 39\% das propriedades estão a uma distância de 350 m dos fragmentos, considerada máxima para que ocorra a conectividade estrutural, e possuem esse tipo de manejo.
\end{abstract}

Palavras-chave: Agrossistemas; Área protegida; Paisagem.

\section{AGRICULTURAL MANAGEMENT IN THE UNA'S WILDLIFE REFUGE: A PERSPECTIVE OF CONSERVATION BY AGROFORESTRY}

\begin{abstract}
This study investigates the types of management and socioeconomic profiles related to those, in the rural properties of the Una's Wildlife Refuge. It has been assumed that the successional agroforestry are the most favorable for the conservation of the Atlantic Rainforest biome, including the landscape scale. Therefore, the objective of this study was to investigate the socio-economic aspects associated with the adoption of a type of management and its integration into the landscape. Several interviews were performed in 73 properties between January and May 2011. Data on agricultural systems were classified into types of management: conventional, traditional, organic and agroforestry. The results indicate the predominance of the traditional type of management in the properties of Una's Wildlife Refuge. The socioeconomic profile found is consistent with the characteristics of the predominant type of management, the traditional. In the traditional management, we observed a greater contribution of family labor and a fewer source of income from rural labor to others. Training for agriculture was the only socioeconomic variable that indicated greater importance for agroforestry. In the landscape, the results pointed the need for adaptation of the practices used in traditional agroecosystems to the biome's conservation goals, because 39\% of the properties are within the distance of $350 \mathrm{~m}$ from the nearest fragment (considered the maximum to occur structural connectivity) and have this type of management.
\end{abstract}

Keywords: Agroecosystems; Protected area; Landscape.

\footnotetext{
${ }^{1}$ Recebido em 05.08.2013 aceito para publicação em 03.12.2013.

${ }^{2}$ Programa de Pós Graduação em Desenvolvimento Regional e Meio ambiente, Universidade Estadual de Santa Cruz, UESC, Brasil. E-mail: <sollberg2@hotmail.com>.

${ }^{3}$ Departamento de Ciências Agrárias e Ambientais, Universidade Estadual de Santa Cruz, UESC, Brasil. E-mail: <aleschi@uesc.br> e<eugeniabruck@hotmail.com>.
} 


\section{INTRODUÇÃO}

Em uma escala de paisagem, os remanescentes do bioma Mata Atlântica estão inseridos em uma matriz composta por um mosaico agrícola-urbano e, portanto, fragmentados (LIRA et al., 2012). Devido ao estado atual de conservação do bioma e à constante pressão das atividades humanas, a criação de Unidades de Conservação (UC), por si só, não garantirá a retenção de populações viáveis nessas áreas, principalmente em fragmentos pequenos, isolados e em diferentes estágios de sucessão florestal (FONSECA et al., 2009; RIBEIRO et al., 2009).

A esse respeito, uma das diretrizes mais aplicadas no planejamento da conservação da biodiversidade é a criação de redes de áreas protegidas conectadas por corredores florestais. Essas ações têm o objetivo de promover uma matriz permeável onde o uso da terra pode coexistir com a biodiversidade florestal (GALINDOLEAL; CÂMARA, 2005; SANDERSON et al., 2003).

Nesse contexto, o Refúgio de Vida Silvestre (REVIS) de Una foi criado (como uma zona-tampão) no entorno da Reserva Biológica (REBIO) de Una. Ambas estão localizadas? na região Sul da Bahia, que está inserida dentro da unidade de gestão territorial para conservação denominada Corredor Central da Mata Atlântica (SCHIAVETTI et al., 2010), mais especificamente no chamado minicorredor Una-Baixão-Lontras (SAVE BRASIL, 2009).

O desafio para gestão do REVIS é o mesmo enfrentado por gestores de outras zonas do entorno de áreas protegidas, que buscam conciliar os objetivos de longo prazo da conservação, com as necessidades imediatas das populações humanas que vivem no local (BUDHATHOKI, 2004).

Neste trabalho, foi adotado como pressuposto que, entre os tipos de agrossistemas, os princípios e práticas adotados naqueles sistemas agroflorestais (SAF) chamados de agroflorestas sucessionais podem ser considerados os mais favoráveis à conservação dos remanescentes de Mata Atlântica. Essa premissa está baseada em diversas publicações (MCNEELY; SCHERR, 2003; SCHROTH et al., 2004; SCHROTH et al., 2011; VIEIRA et al., 2009), que apontam a característica dos SAFs de aliar a coexistência e interação do uso da terra com a conservação. Reconhecem que, com a utilização de SAFs, a necessidade de abertura de novas áreas para a agricultura pode diminuir, pois as práticas utilizadas nesses sistemas são favoráveis à conservação dos recursos naturais, como o solo (CAMPANHA et al., 2007), os recursos hídricos, entre outros; podem servir de suporte à conservação da biodiversidade biológica e para a recuperação de áreas degradadas (SOUZA; PIÑA-RODRIGUES, 2013).

Segundo Tabarelli et al. (2010), os SAFs, apesar de não substituírem as áreas florestais, são os sistemas agrícolas que oferecem melhores condições para a conservação da biodiversidade através da viabilização da conectividade entre áreas florestais. Trabalhos realizados na Mata Atlântica da Região Cacaueira do Sul da Bahia (CASSANO et al., 2009; MCNEELY; SCHROTH, 2006; PARDINI et al., 2009) citaram os SAFs como exemplo de uma das estratégias utilizadas para aumentar os efeitos benéficos da permeabilidade da matriz.

Por existir grande diversidade de SAFs, encontram-se variações no potencial para conservação de cada agrossistema, de acordo com o seu grau de complexidade. Desde sistemas simples de consórcio, de um cultivo com uma espécie arbórea, até sistemas mais funcionais para conservação, as agroflorestas sucessionais (SCHROTH; HARVEY, 2007). Esses são apontados como sistemas produtivos que buscam imitar as funções ecológicas, encontradas nos ecossistemas naturais (SCHROTH et al., 2004; VIEIRA et al., 2009), pois possuem maior diversidade de espécies; arranjo espacial, temporal e estratificação que imitam a sucessão natural (estrato baixo, médio, médio-alto, alto e emergente), além de incluírem espécies nativas.

No Sul da Bahia, desde o século XVIII tem-se registros de um tipo de sistema agroflorestal. Até hoje, esse sistema é característica marcante da paisagem da região, conhecido como cabruca. Consiste no cultivo do cacau (Theobroma cacao) à sombra do dossel da floresta nativa (ARAUJO et al., 1998; JOHNS, 1999; SAMBUICHI et al., 2012). Esse sistema de cultivo do cacau (graças à tolerância da espécie à sombra) é considerado amigável para a vida silvestre (CASSANO et al., 2009; SCHROTH, 2011), pois apresenta superioridade em termos de conservação da biodiversidade, quando comparado com outras plantações tropicais (monoculturas de dendê, seringa ou café), agricultura ou pastagens (RICE; GREENBERG, 2000; SCHROTH et al., 2004). É necessário ressaltar 
que esse sistema foi adotado como sistema de produção, antes de existir como tal a preocupação com a conservação da biodiversidade. Portanto, no histórico do cultivo na região, esse caráter favorável à conservação foi afetado de acordo com aspectos socioeconômicos, como oscilações de mercado e tendências de práticas agronômicas (JOHNS, 1999).

Segundo Schroth et al. (2004), além da estrutura, composição e tipo de manejo dos agrossistemas, sua posição na paisagem também é fator determinante para seu valor como possível corredor, hábitat, ou função de amortecimento das bordas dos fragmentos. Afirmaram que, na escala da paisagem, os sistemas agroflorestais complexos podem promover hábitat secundário para espécies que são tolerantes a um grau de distúrbio; contribuir para reduzir a conversão de hábitat natural; e podem colaborar para manutenção dos remanescentes e conservação de suas populações, através da criação de uma matriz mais permeável.

A fim de conhecer a atual situação das propriedades existentes no REVIS de Una, os agrossistemas pesquisados tiveram suas práticas classificadas em quatro tipos de manejo: do convencional, o mais desfavorável para a conservação, passando pelo tradicional, orgânico até as agroflorestas sucessionais. Foram investigados o perfil socioeconômico e variações de suas características em relação aos tipos de manejo. $\mathrm{Na}$ escala de paisagem, a distância entre os agrossistemas, caracterizados pelo tipo de manejo, e os fragmentos remanescentes foi calculada, a fim de avaliar seu potencial para conectividade estrutural.

\section{MATERIAL E MÉTODOS}

\section{1. Área de estudo}

A área de estudo inclui a área do REVIS (23.404 ha) e da REBIO de Una (18.515 ha). Para análise da paisagem, além dos fragmentos florestais inseridos nessas áreas, foram considerados também aqueles que distam até $5 \mathrm{~km}$ da REBIO. O REVIS de Una foi criado através de Decreto Presidencial (de 21 de dezembro de 2007), abrangendo uma faixa costeira com áreas de restinga, manguezal e áreas de floresta da Mata Atlântica (CASTILHO et al., no prelo). A área situa-se na região Sul da Bahia e está inserida no Município de Una, com pequena parte dentro do Município de Ilhéus. Segundo o IBGE (2010), as principais lavouras permanentes, considerando o total de área plantada, são: de cacau, com 16.800 ha plantados; de seringa, com 5.000 ha; e de dendê, com 2.600 ha. A principal lavoura temporária é a de mandioca, com 950 ha de área plantada.

\subsection{Coleta de dados}

O estudo foi baseado em dados primários coletados por meio de entrevistas estruturadas (DENCKER, 1998). A coleta de dados de campo foi realizada em uma amostra de $26 \%$ das propriedades (ICMBio) do REVIS de Una, entre janeiro e maio de 2011, com 73 propriedades (Figura 1).

Os principais aspectos abordados nas entrevistas foram: perfil socioeconômico do entrevistado e da propriedade; e características e práticas da agricultura, que foram classificadas em quatro tipos de manejo (ABREU, 1994; GLIESSMAN, 2001; REYES et al., 2005):

a) Convencional, quando não possui: sucessão natural, estratificação, insumos orgânicos, produção própria de adubo (esterco, biomassa), produção própria de sementes (dentro da propriedade, sem depender da compra de sementes), mão de obra familiar e consumo da produção. Faz uso de maquinário e irrigação.

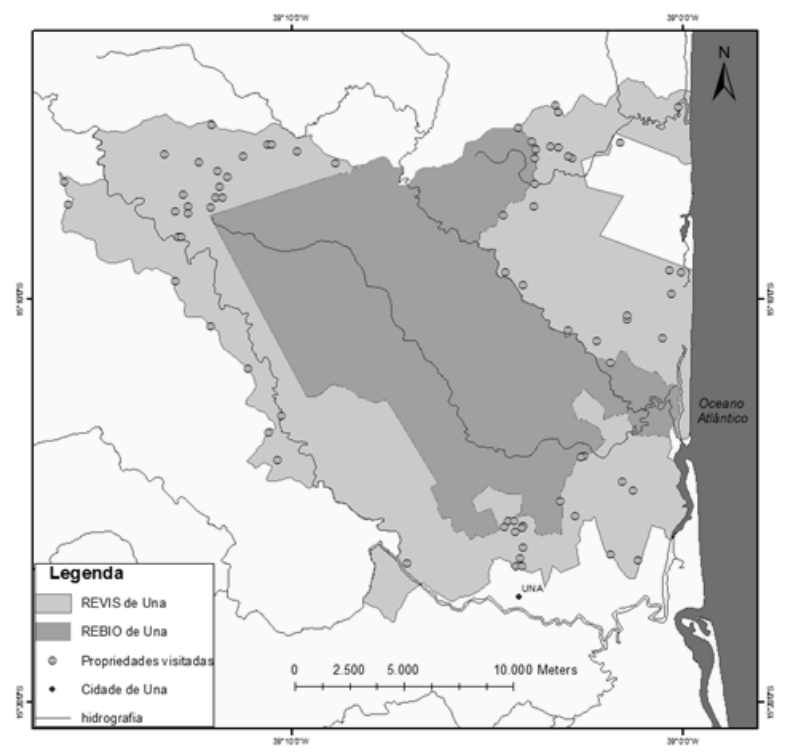

Figura 1 - Localização das propriedades do REVIS Una avaliadas quanto ao sistema de manejo agrícola.

Figure 1 -Location of the properties from Una's Wildlife Refuge evaluated by agricultural management system.

Revista Árvore, Viçosa-MG, v.38, n.2, p.241-250, 2014 
b) Tradicional, quando realiza rotação de culturas, rotação de terras, corte e queima, consórcio; possui: diversidade de cultivos, adubo e sementes próprios, mão de obra familiar e consumo da produção. Não utiliza maquinário.

c) Orgânico, quando apenas utiliza insumos orgânicos, realiza rotação de culturas, aproveita biomassa, pode utilizar semente própria ou do mercado desde que orgânica, mão de obra familiar, ou contratada. Utiliza maquinário ou não.

d) Agroflorestal, quando não utiliza corte-e-queima; usa: consórcio, sucessão natural, aproveitamento de biomassa, espécie funcional (espécie que desempenha pelo menos uma função protetiva no sistema: sombreamento, quebra-ventos, refúgio, conservação do solo, entre outros. Pode desempenhar apenas função protetiva ou, além desta, alguma função produtiva alimento, madeira, forragem), diversidade de cultivos, espécie arbórea e estratificação. Utiliza apenas insumos orgânicos e adubo próprio.

\subsection{Análise}

As análises estatísticas foram realizadas no software BioStat, através de testes de qui- quadrado, para verificar a variação das características socioeconômicas, de acordo com as classes de tipos de manejo das propriedades do REVIS. O teste de qui-quadrado de partição revelou qual compartimento específico colaborou mais para variação. As variáveis que apresentaram "p" inferior ou igual a 0,05 foram incluídas na análise que utilizou a regressão logística múltipla para verificar a influência dos aspectos socioeconômicos em relação à adoção dos tipos de manejo. Para cada tipo de manejo, foi feita a regressão logística, sendo a variável resposta a classe de manejo (BUYINZA et al., 2007).

Para análise da paisagem em Sistema de Informações Geográficas (SIG), os pontos de coordenadas geográficas coletados com GPS, referentes à localização das propriedades, foram plotados no software Arc. GIS 10. Os fragmentos considerados foram aqueles dentro da REBIO e do REVIS e que distavam até $5 \mathrm{~km}$ da REBIO do shape na escala de 1 para 50 mil (1:50.000) m (SOS MATA ATLÂNTICA/INPE, 2002).

O cálculo da distância de cada propriedade (caracterizada pelo respectivo tipo de manejo), em relação ao fragmento mais próximo, foi realizado no Arc. GIS
10 (função do Arc. toolbox; Analysis tools; Proximity; e Near). Na paisagem, as propriedades localizadas a até $350 \mathrm{~m}$ de algum fragmento foram consideradas potenciais para a conservação, já que esta é a distância máxima que a maioria das espécies consegue se locomover em áreas abertas adjacentes à floresta (PIRES et al., 2004).

\section{RESULTADOS}

O perfil socioeconômico dos entrevistados (entre proprietários, responsáveis, membros da família ou trabalhadores) revela que a média de idade é de 49 anos; a maior parte dos entrevistados (78\%) é do gênero masculino e apenas 15\% deles já receberam algum tipo de capacitação relacionada à atividade agronômica. A maioria não possui escolaridade (48,2\%) e tem como principal fonte de renda recursos gerados da atividade na propriedade rural (43,8\%); 54,8\% declararam que estão na propriedade há mais de 10 anos, seja como moradores, trabalhadores ou proprietários.

As atividades econômicas mais citadas foram a agricultura (em 88\% das propriedades), seguida do extrativismo e, ou, silvicultura (71\% das propriedades). Além dessas atividades, 56\% das propriedades destinamse também a algum tipo de moradia. Essas duas atividades econômicas (agricultura, extrativismo/silvicultura) e a função de moradia foram encontradas de forma concomitante em 36\% das propriedades.

Entre as propriedades pesquisadas, apenas 29\% participaram de alguma instituição de organização social, como associação, cooperativa; e o mesmo percentual foi calculado para aqueles que afirmaram que recebiam assistência técnica.

A respeito da variedade de cultivos, o da seringueira foi o mais citado, encontrado em 75\% das propriedades, seguido pelo cultivo do cacau (73\%), da mandioca (40\%) e do coco (37\%). A percepção dos produtores sobre a qualidade do solo revela que os entrevistados consideram o solo regular (47\%) e com aptidão para algum tipo de cultivo (74\%). O cultivo da seringueira ( $n=25$ ) foi o que obteve maior número de indicações como adequado ao solo do local, seguido pela mandioca $(n=12)$ e pelo cacau e pelo coco $(n=9)$.

Os resultados agroeconômicos apontaram que o beneficiamento da produção é realizado em $65 \%$ das propriedades e a transformação, em apenas 26\%. A 
principal forma de comercialização da produção é por meio dos atravessadores (60\%). Apenas 25\% dos entrevistados enfrentavam algum tipo de dificuldade para comercializar sua produção. Poucos (19\%) contavam com fonte de financiamento de sua produção.

Sobre os meios de produção e infraestrutura, apenas $12 \%$ utilizavam maquinário, enquanto $53 \%$ faziam uso da força animal. Itens de infraestrutura (sistemas de captação ou bombeamento de água, casa de farinha ou barcaça etc.) foram encontrados em 31\% das propriedades e $4 \%$ possuíam sistema de irrigação. Apenas $18 \%$ das propriedades são servidas pela companhia estadual de energia. A lenha foi apontada como principal fonte combustível utilizada nas propriedades (75\%), seguida da gasolina ou do óleo diesel (22\%).

A classificação de tipos de manejo revelou que $60 \%(n=44)$ dos agroecossistemas possuíam o manejo “Tradicional”, seguido de $21 \%(n=15)$ com manejo “Convencional”. Os manejos “Orgânico" e “Agroflorestal” apresentaram apenas 8\% $(n=6)$ e 7\% $(\mathrm{n}=5)$ do total, respectivamente. Três propriedades não foram incluídas na classificação, pois não apresentavam qualquer tipo de atividade agropecuária (Figura 2).

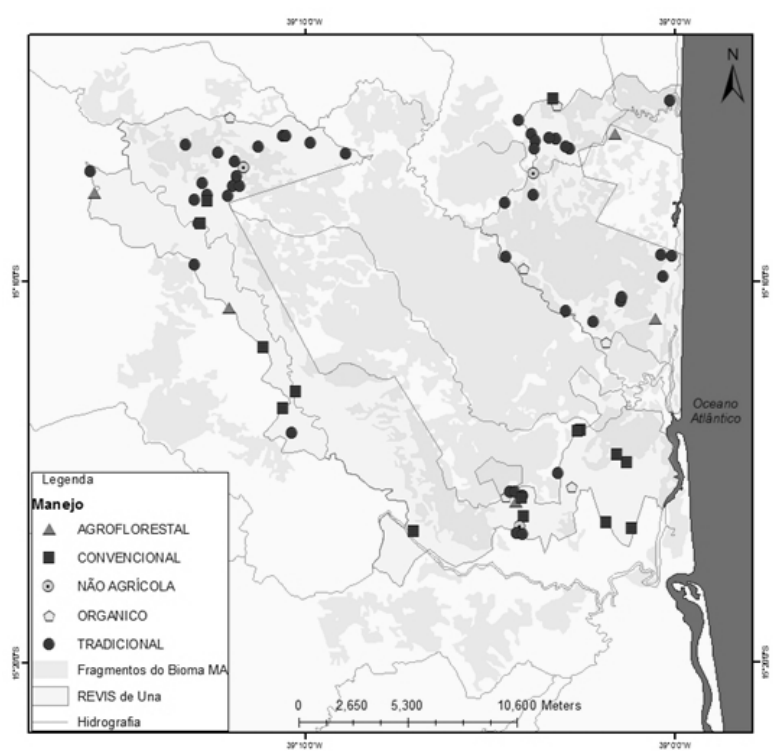

Figura 2 - Mapa de localização das classificações de tipos de manejo nas propriedades analisadas no REVIS de Una.

Figure 2-Location map of the types of management classification on the properties studied.
Quanto às práticas agronômicas, apenas 10\% dos agrossistemas pesquisados apresentavam o uso da prática de aproveitamento de biomassa, 39\% faziam uso de espécies funcionais e $27 \%$ utilizavam corte e queima.

Os aspectos socioeconômicos analisados apresentaram variação significativa, de acordo com os tipos de manejo (Tabela 1).

A mão de obra familiar e o tipo de fonte de renda apresentaram variação específica nas classes de manejo "tradicional”, quando comparado com "convencional” e “orgânico”. A capacitação foi uma variável significativa apenas no manejo "agroflorestal”.

A variável “tempo na propriedade”, apesar de não apresentar variação geral significativa $(p=0,1112)$, mostrou variação significativa ( $p=0,0021)$ em um conjunto de dados específicos, nas classes "convencional” e “tradicional”, especificamente na categoria "mais de 10 anos na propriedade”.

Dos resultados de tipos de manejo em relação à paisagem (Tabela 2), destaca-se que 58\% das propriedades estavam até 350 m de distância de algum fragmento; dessas, 39\% possuíam manejo “tradicional”, 9\% “convencional” e apenas 6\% possuíam o manejo “orgânico” e 4\% o “agroflorestal”.

A análise da conectividade estrutural da paisagem (Figura 3) revela que, dos 76 fragmentos, 21 (28\%) estavam entre 75 e 100 m de distância de algum outro fragmento (Figura 3A). Quanto à classe de tamanho, a maioria (28 fragmentos) tinha entre 10 e 50 ha (Figura 3B).

\section{DISCUSSÃO}

Os agrossistemas analisados não se enquadravam perfeitamente nas classificações de tipos de manejo determinadas pelos conceitos teóricos das classes definidas neste trabalho: convencional, tradicional, orgânico e agroflorestal.

Segundo Tscharntkea et al. (2012), a dicotomia convencional versus agroecológico é simplista, pois muitas outras variações são encontradas entre essas categorias. Porém, algumas tendências podem ser observadas, de acordo com o tipo de manejo. A agricultura convencional, por exemplo, geralmente depende de grandes investimentos em insumos, enquanto a agricultura de baixo uso de insumos está associada

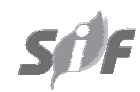

Revista Árvore, Viçosa-MG, v.38, n.2, p.241-250, 2014 
Tabela 1 - Variações dos aspectos socioeconômicos de acordo com os tipos de manejo.

Table 1 - Changes in socioeconomic aspects according to the types of management.

\begin{tabular}{|c|c|c|c|c|c|}
\hline $\begin{array}{c}\text { Tipo de manejo } \\
\text { Variável }\end{array}$ & $\begin{array}{c}\text { Total } \\
(\mathrm{N}=70) \\
\text { N. }(\%)\end{array}$ & $\begin{array}{c}\text { Convencional } \\
(\mathrm{n}=15) \\
\text { N. }(\%)\end{array}$ & $\begin{array}{l}\text { Tradicional } \\
(\mathrm{n}=44) \\
\text { N. }(\%)\end{array}$ & $\begin{array}{l}\text { Orgânico } \\
(\mathrm{n}=6) \\
\text { N. (\%) }\end{array}$ & $\begin{array}{c}\text { Agroflorestal } \\
(\mathrm{n}=5) \\
\text { N. (\%) } \\
\end{array}$ \\
\hline \multicolumn{6}{|l|}{$\overline{\text { MDOF* }}$} \\
\hline Sim & $42(60)$ & $1(7)$ & $37(84)$ & $1(17)$ & $3(60)$ \\
\hline Não & $28(40)$ & $14(93)$ & 7 (5) & $5(83)$ & $2(40)$ \\
\hline \multicolumn{6}{|l|}{ TFR* } \\
\hline Não agrícola & $17(24)$ & $0(0)$ & $15(34)$ & $1(17)$ & $1(20)$ \\
\hline Da propriedade & $32(46)$ & $4(27)$ & $24(55)$ & $0(0)$ & $4(80)$ \\
\hline Trabalhador rural & $21(30)$ & 11 (73) & $5(11)$ & $5(83)$ & $0(0)$ \\
\hline \multicolumn{6}{|l|}{$\mathrm{CAP}^{* *}$} \\
\hline Sim & 12 (17) & $2(13)$ & 7 (16) & $0(0)$ & $3(60)$ \\
\hline Não & $58(83)$ & $13(87)$ & $37(84)$ & $6(100)$ & $2(40)$ \\
\hline \multicolumn{6}{|l|}{ MOR* } \\
\hline Sim & 41 (59) & $0(0)$ & $37(84)$ & $1(17)$ & $3(60)$ \\
\hline Não & $29(41)$ & $15(100)$ & $7(16)$ & $5(83)$ & $2(40)$ \\
\hline \multicolumn{6}{|l|}{ AGR* } \\
\hline Sim & $64(91)$ & $15(100)$ & $43(98)$ & $1(17)$ & $5(100)$ \\
\hline Não & $6(9)$ & $0(0)$ & $1(22)$ & $5(83)$ & $0(0)$ \\
\hline \multicolumn{6}{|l|}{ PEC* } \\
\hline Sim & $11(16)$ & $2(13)$ & $3(7)$ & $6(100)$ & $0(0)$ \\
\hline Não & $59(84)$ & $13(87)$ & 41 (93) & $0(0)$ & $5(100)$ \\
\hline
\end{tabular}

Nota: ${ }^{*}<<0,0001 ;{ }^{*} \mathrm{p}=0,048 ;$ MDOF = mão de obra familiar, TFR = tipo de fonte de renda; CAP = capacitação; MOR = moradia; AGR = agricultura; e PEC = pecuária.

Note: $* p<0.0001 ; * * p=0.048 ; M D O F=$ family labor, TFR = source type of income; CAP = training; MOR = house;

$A G R=$ agriculture; and $P E C=$ livestock.

Tabela 2 - Distância dos tipos de manejo (propriedades) ao fragmento mais próximo.

Table 2 - Distance of the type of management (properties) to the nearest fragment.

\begin{tabular}{|c|c|c|c|c|c|c|c|c|c|c|}
\hline Distância (m) & Total (n. prop.) & $\%$ & CONV & $\%$ & TRAD & $\%$ & ORG & $\%$ & AGRO & $\%$ \\
\hline $0-350$ & 40 & $58 \%$ & 6 & $9 \%$ & 27 & $39 \%$ & 4 & $6 \%$ & 3 & $4 \%$ \\
\hline $350-\mid 1000$ & 21 & $30 \%$ & 4 & $27 \%$ & 14 & $32 \%$ & 2 & $33 \%$ & 1 & $20 \%$ \\
\hline$>1000$ & 9 & $13 \%$ & 5 & $33 \%$ & 3 & $7 \%$ & 0 & $0 \%$ & 1 & $20 \%$ \\
\hline Total & 70 & $100 \%$ & 15 & $100 \%$ & 44 & $100 \%$ & 6 & $100 \%$ & 5 & $100 \%$ \\
\hline
\end{tabular}
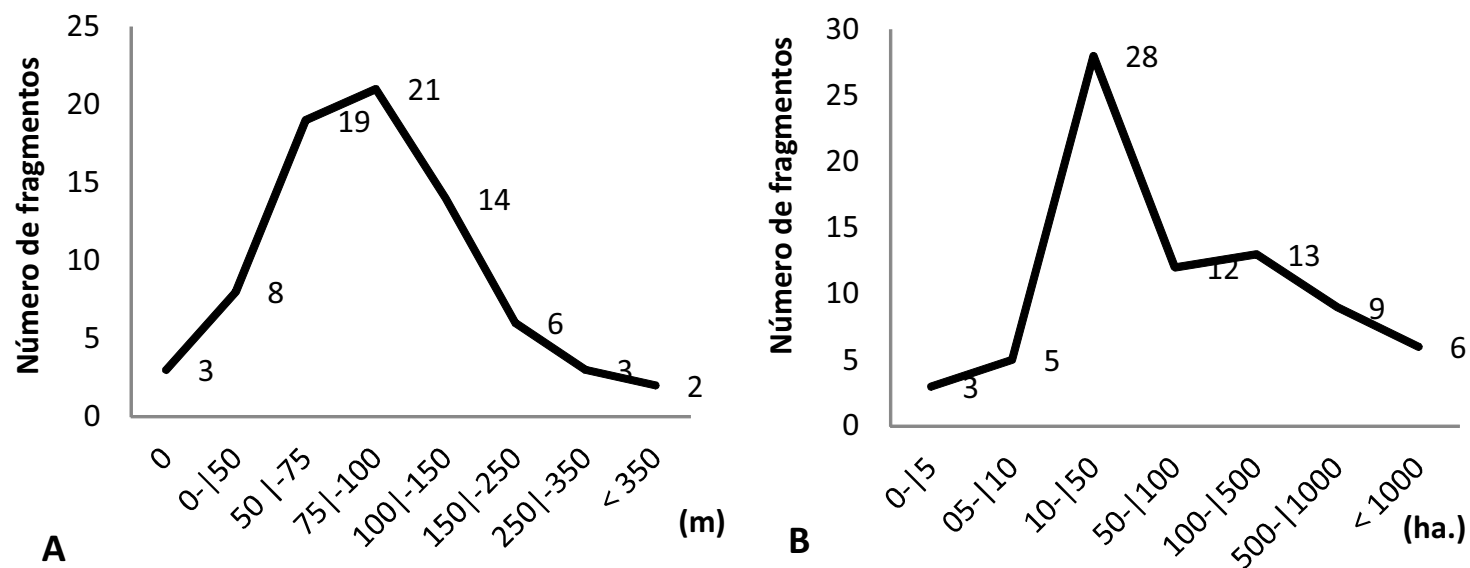

Figura 3 - Número de fragmentos por classes de grau de isolamento (A) e por classes de tamanho (B) dos fragmentos analisados. Figure 3 - Number of fragments by class and degree of isolation (A) and by size (B) of the analyzed fragments.

Revista Árvore, Viçosa-MG, v.38, n.2, p.241-250, 2014 
a populações com menos recursos financeiros que dependem mais da biodiversidade e das interações ecológicas.

O principal tipo de manejo encontrado, entre os agrossistemas pesquisados no REVIS de Una, foi o tradicional. Nesse caso, é esperada a dependência dos agricultores em relação aos recursos das florestas nativas adjacentes. Na pesquisa sobre práticas agroflorestais no entorno de um Parque Nacional em Uganda, foi encontrado alto percentual de consumo de lenha (BUYINZA et al., 2007), assim como nos resultados desta pesquisa. Os resultados da análise da paisagem reforçam essa característica, já que os agrossistemas em contato direto com os remanescentes utilizavam o manejo tradicional, em sua maioria, enquanto o manejo convencional só foi encontrado em propriedades há mais de $50 \mathrm{~m}$ de algum remanescente.

O perfil socioeconômico encontrado nas propriedades que possuíam manejo tradicional está de acordo com o descrito na literatura (ABREU, 1994; GLIESSMAN, 2001; REYES et al., 2005). Esse perfil pode limitar a adoção de sistemas agroflorestais. Buyinza et al. (2007) afirmaram que treinamento e educação formal e informal têm potencial para aumentar a taxa de adoção de novas técnicas agrícolas, como as agroflorestais. Além disso, apontaram para a tendência de as pessoas que não possuíam escolaridade não adotarem práticas agroflorestais. No estudo de Reyes et al. (2005) sobre as florestas tropicais da Tanzânia a respeito da comparação de aspectos socioeconômicos entre as práticas tradicionais e agroflorestais, foi destacado que o fato de a maioria dos agricultores saber ler e escrever muito contribuiu para a compreensão de conteúdos de melhoria de técnicas agrícolas. Essa relação pôde ser verificada nas variações socioeconômicas observadas no tipo de manejo agroflorestal, em que a maioria possuía capacitação técnica, ao contrário dos outros tipos de manejo.

Segundo Reyes et al. (2005), em estudo comparativo entre sistemas agroflorestais e tradicionais na Tanzânia, atividades de extensão rural são necessárias para fornecer informações básicas, como utilização e preparação de compostos, já que muitos agricultores ainda removem a biomassa e a queimam. Essa afirmação pode ser aplicada na situação do REVIS, pois a minoria dos entrevistados aproveitava a biomassa, assim como práticas de adubação verde, diversificação de cultivos, entre outras que poderiam ser adotadas.
Os resultados agroeconômicos sobre as culturas, como os cultivos mais citados, são compatíveis com os dados oficiais da região (IBGE, 2010) e corroboram os resultados de qualidade do solo e de cultivos citados como adequados ao local. Os cultivos mais citados como aptos para o solo local são justamente aqueles adaptados a solos ácidos ou moderadamente ácidos (EDEN; ANDRADE, 1987; LAL, 1995), o que corrobora o resultado sobre a qualidade dos solos que foi apontada como "regular".

Esta pesquisa revelou baixa participação em organizações sociais, entre os entrevistados. Segundo Ashley et al. (2006), o fortalecimento de organizações sociais é considerado quase um pré-requisito para que os sistemas agroflorestais sejam bem-sucedidos. Reyes et al. (2005) afirmaram que associações de agricultores fortes podem contribuir para a melhoria da infraestrutura de comercialização, como transporte rural. Experiências bem- sucedidas de cooperativas também mostraram a diminuição da insegurança em relação às variações de preços.

Somadas a essas características, o perfil da produção indica baixo acesso à infraestrutura. Apesar de o beneficiamento da produção ser realizado em mais da metade das propriedades, ele está diretamente associado à produção do cacau (presente na maioria das propriedades), que exige um processo de secagem e, por vezes, a fermentação da amêndoa, porém não significa uma valorização da produção. Uma tendência atual de valorização da produção cacaueira na região é a certificação orgânica (NIESTEN et al., 2004), porém nenhuma propriedade pesquisada possui qualquer tipo de certificação.

A ocorrência de transformação da produção está associada ao cultivo da mandioca para produção de farinha, que conta com infraestrutura rudimentar. Uma minoria utilizava maquinário e infraestrutura (sistemas de captação ou bombeamento de água, casa de farinha ou barcaça etc.), acesso à eletricidade da companhia de energia estadual e uso de combustível fóssil. A maioria utiliza tração animal e lenha; essas características também são esperadas na agricultura tradicional.

Segundo Reyes et al. (2005), o baixo acesso, ou o acesso inadequado ao crédito, ou financiamento capital, é considerado um dos principais limitantes para o aumento da produção e renda familiar entre agricultores das florestas tropicais na Tanzânia. Admitiram que, por

Revista Árvore, Viçosa-MG, v.38, n.2, p.241-250, 2014 
vezes, devido à falta de recursos para sustento da família, foram impelidos a aumentar a exploração dos recursos florestais. Porém, é importante ressaltar que as possíveis políticas públicas para melhorar o acesso ao mercado, às fontes de financiamento e à melhoria de infraestrutura têm impactos ambíguos, que podem estimular ou diminuir a conversão de florestas tropicais (PRIESS, 2007).

Apenas 4\% do total das propriedades pesquisadas possuíam manejo agroflorestal e estavam à distância de até 350 m de algum fragmento. Essas propriedades possuíam proximidade e manejos favoráveis para fins de conservação, portanto podem, inclusive, desempenhar o papel de trampolins, entre os fragmentos (RANTA et al., 1998). Dos 9\% das propriedades que estão conectadas aos fragmentos, $7 \%$ tinham manejo tradicional e 2\%, orgânico. Propriedades com manejo agroflorestal adjacentes aos fragmentos poderiam amenizar os efeitos de borda (SCHORTH et al., 2004).

A existência de remanescentes florestais próximos a agroecossistemas, bem como a existência de sistemas agroflorestais, pode ser considerada favorável tanto para a produtividade dos cultivos quanto para a sua conservação. Priess (2007) apresentou dados empíricos dos efeitos negativos do aumento da distância, entre as áreas de floresta e as áreas de cultivo de café, sobre a diversidade de polinizadores e frutificação do café nas florestas tropicais do Sulawesi, na Indonésia. Johns (1999) afirmou que as áreas remanescentes adjacentes aos agroecossistemas fornecem serviços ambientais, como: polinização, controle de pragas, barreiras de vento, interações com clima e recursos hídricos, entre outros, serviços esses que também podem ser promovidos pelos sistemas agroflorestais.

A maioria (63\%) dos fragmentos analisados apresentou área menor que 100 ha. Segundo Bierregaard e Dale (1996), é o tamanho mínimo de um fragmento florestal para que este possa sustentar uma proporção considerável de espécies de florestas tropicais (excluindo grandes mamíferos).

Portanto, o manejo do REVIS, uma UC que permite a atividade agrícola desde que compatível com seus objetivos de conservação, deve incentivar os SAFs existentes e promover a transição agroecológica nos agrossistemas tradicionais que distam até $350 \mathrm{~m}$ de algum fragmento remanescente (39\%), pois esses agrossistemas possuem potencial para favorecer a conectividade florestal devido à sua localização na paisagem. Porém, devido à complexidade de gestão dos sistemas agroflorestais nos aspectos agronômicos, econômicos, sociais e ecológicos, a baixa escolaridade e a falta de capacitação são limitantes para uma transição de sistemas convencionais e tradicionais para sistemas agroflorestais (FISCHER; VASSEUR, 2000).

\section{CONCLUSÃO}

O manejo tradicional, encontrado na maioria das propriedades pesquisadas, apesar de não ser o ideal, apresentou variações similares ao manejo agroflorestal, em características favoráveis à conservação, como: adoção de práticas de consórcio e sucessão natural. Além disso, apresentou variação significativa na prática de produção de sementes no manejo tradicional, que não foi encontrada, nem mesmo no manejo agroflorestal.

Para que o REVIS desempenhe seu papel na conservação da Mata Atlântica, o plano de manejo da UC deve, portanto, incluir a criação de políticas públicas voltadas para a adequação das práticas dos agrossistemas aos objetivos da conservação, através de programas de incentivo à transição agroecológica.

Para promover a transição agroecológica dentro das propriedades do REVIS, é necessária a melhoria em educação, capacitação, acesso a incentivos econômicos, fomentar a criação e o fortalecimento das organizações sociais, bem como desenvolver atividades de extensão e apoio técnico focados em metodologias participativas de construção do conhecimento, buscando a efetividade e continuidade da adoção de práticas agroflorestais pelos produtores.

\section{REFERENCIAS}

ABREU, L. S. Impactos sociais e ambientais na agricultura: uma abordagem histórica de um estudo de caso. Brasília: Embrapa-SPI, 1994. p.17-43

ARAUJO, M. et al. A Mata Atlântica no Sul da Bahia. Série Cadernos da Reserva da Biosfera da Mata Atlântica, n. 8, p.35, 1998.

ASHLEY, R.; RUSSELL, D.; SWALLOW, B. The policy terrain in protected area landscapes: challenges for agroforestry in integrated landscape conservation. Biodiversity \& Conservation, v.4, n.2 p.663-689, 2006. 
BIERREGAARD, R.O.; DALE, V.H. Islands in an ever-changing sea: the ecological and socioeconomic dynamics of Amazonian rainforests fragments. In: SCHELLAS, J.; GREENBERG, R. (Ed.). Forest patches in tropical landscapes. Washington: University Island Press, 1996. p.187-204.

BUDHATHOKI, P. Linking communities with conservation in developing countries: buffer zone management initiatives in Nepal. Oryx, v.38, n.3, p.334-341, 2004.

BUYINZA, M.; KABOGGOZA, J.R.; NABALEGWA, M. Agroforestry practices in the buffer zone area of Mt Elgon National Park, Eastern Uganda. African Journal of Ecology, v. 45, n.1, p.48-53, 2007.

CAMPANHA, M. M. et al. Análise comparativa das características da serrapilheira e do solo em cafezais (coffea arabica l.) Cultivados em sistema Agroflorestal e em monocultura, na zona da mata MG. Revista Árvore, v.31, n.5, p.805-812, 2007.

CASSANO, C. R. et al. Landscape and farm scale management to enhance biodiversity conservation in the cocoa producing region of southern Bahia, Brazil. Biodiversity and Conservation, v.18, n.3, p.577-603, 2009.

CASTILHO, L. C. et al. Knowledge on and threats to Chaetomyssubspinosus (Rodentia:

Erethizontidae) within protected areas in the Atlantic Forest, Brazil. Tropical

Conservation Science, v.6, n.6, p.796-810, 2013.

DENCKER, A. Métodos e técnicas de pesquisa em turismo. São Paulo: Futura, 1998. p.137-157.

EDEN, M. J.; ANDRADE, A. Ecological aspects of swidden cultivation among the Andoke and Witoto Indians of the Colombian Amazon. Human Ecology, v.15, n.3, p.339-359, 1987.

FISCHER, A.; VASSEUR, L. The crisis in shifting cultivation practices and the promise of agroforestry: a review of the Panamanian experience. Biodiversity and Conservation, v.9, n.6, p.739-756, 2000.
FONSECA, C. R. et al. Towards an ecologically sustainable forestry in the Atlantic Forest. Biological Conservation, v.142, n.1, p.1144-1154, 2009.

GALINDO-LEAL, C.; CÂMARA, I. G. Mata Atlântica - Biodiversidade, ameaças e perspectivas. São Paulo: Fundação SOS Mata Atlântica - Belo Horizonte: Conservação Internacional, 2005. p.459-465.

GLiessman, S. R. Agroecologia: processos ecológicos em agricultura sustentável. 2.ed. Porto Alegre: Universidade Federal do Rio Grande do Sul, 2001. p.3-30.

INSTITUTO BRASILEIRO DE GEOGRAFIA E ESTATÍSTICA - IBGE. Disponível em: <http:// censo2010.ibge.gov.br/>

ICMBio. REVIS. Refúgio de Vida Silvestre. 2010. (Informativo.MMA)

JOHNS, N. Conservation in Brazil's chocolate forest: the unlikely persistence of the tradidional cocoa agroecosystem. Environmental Management, v.23, n.1, p.31-47, 1999.

LAL, R. Sustainable management of soil resources in the humid tropics. Tokyo: The United Nations University, 1995. p.20-23.

LIRA, P. K. Land-use and land-cover change in Atlantic Forest landscapes. Forest Ecology and Management, v.278, n.1, p.80-89, 2012.

MCNEELY, J. A.; SCHERR, S. Ecoagriculture: strategies to feed the world and save wild biodiversity. Washington: Island Press, 2003. p. 177-200.

MCNEELY, J. A.; SCHROTH, G. Agroforestry and biodiversity conservation - traditional practices, present dynamics, and lessons for the future.

Biodiversity and Conservation, v.15, n.2, p.549-554, 2006.

NIESTEN, E.T. et al. Commodities and conservation: the need for greater habitat protection in the tropics. Conservation International, Washington, D.C., USA. 2004. p.15-17.

Revista Árvore, Viçosa-MG, v.38, n.2, p.241-250, 2014 
PARDINI, R. et al. The challenge of maintaining Atlantic forest biodiversity: A multi-taxa conservation assessment of specialist and generalist species in an agro-forestry mosaic in southern Bahia. Biological Conservation, v.142, n.6, p.1178-1190, 2009.

PIRES, A. M. Z. C. R. et al. Avaliação da integridade ecológica em bacias hidrográficas. In: SANTOS, J. E.; CAVALHEIRO, F. Faces da polissemia da paisagem: ecologia, planejamento e percepção. São Carlos: Rima, 2004. p.123-150.

PRIESS, J. A. Linking deforestation scenarios to pollination services and economic returns in coffee agroforestry systems. Ecological Applications, v.17, n.2, p.407-417, 2007.

RANTA, P. et al. The fragmented Atlantic rain Forest of Brazil: size, shape and distribution of Forest fragments. Biodiversity and Conservation, v.7, n.3, p.385-403, 1998.

REYES, T.; QUIROZ, R.; MSIKULA, S. Socioeconomic comparison between traditional and improved cultivation methods in agroforestry systems, East Usambara Mountains, Tanzania. Environmental Management, v.36, n.5, p.682-690, 2005.

RIBEIRO, M. C. et al. The brazilian atlantic forest: how much is left, and how is the remaining forest distributed? Implications for conservation.

Biological Conservation, v.142, n.6, p.11411153, 2009.

RICE, R. A.; GREENBERG, R. Cacao cultivation and the conservation of biological diversity. Ambio, v.29, p.167-173, 2000.

SAMBUICHI, R. et al. Cabruca agroforests in southern Bahia, Brazil: tree component, management practices and tree species conservation.

Biodiversity and Conservation, 21:10551077, 2012.

SANDERSON, J. et al. Biodiversity conservation corridors: Planning, implementing, and monitoring sustainable landscapes. Washington, DC: Center for Applied Biodiversity Science, Conservation International, 2003. p.10-11.
SAVE BRASIL; IESB; BIRDLIFEINTERNATIONAL. Complexo de Serras das Lontras e

Una, Bahia: elementos naturais e aspectos de sua conservação. São Paulo: 2009. p.52-55.

SCHIAVETTI, A. et al. Análise das reservas particulares do patrimônio natural como estratégia de conservação na região cacaueira do Sul da Bahia, Brasil. Revista Árvore, v.34, n.4, p.699-711, 2010.

SCHROTH, G. et al. (Ed.) Agroforestry and biodiversity conservation in tropical landscapes. Washington: Island Press, 2004. p.151-430.

SCHROTH, G.; HARVEY, C. Biodiversity conservation in cocoa production landscapes: an overview. Biodiversity and Conservation, v.16, n.8, p.2237-2244, 2007.

SCHROTH, G. et al. Conservation in tropical landscape mosaics: the case of the cacao landscape of southern Bahia, Brazil.

Biodiversity and Conservation, v.20, n.8, p.1635-1654, 2011.

SOS Mata Atlântica, Disponível em: http:// www.sosmatatlantica.org.br/ index.php?section=info\&action=unidades $>$ Acesso em: 15 de junho de 2010.

SOUZA, M. C. S.; PIÑA-RODRIGUES, F. C. M. Desenvolvimento de espécies arbóreas em sistemas agroflorestais para recuperação de áreas degradadas na floresta ombrófila densa, Paraty, RJ. Revista Árvore, v.37, n.1, p.89-98, 2013.

TABARELLI, M. et al. Prospects for biodiversity conservation in the Atlantic Forest: lessons from aging human-modified landscapes. Biological Conservation, v.143, n.10, p.2328-2340, 2010.

TSCHARNTKEA, T. et al. Global food security, biodiversity conservation and the future of agricultural intensification. Biological Conservation, v.151, n.1, p.53-59, 2012.

UPHOFF, N. Grassroots organizations and NGOs in rural development: opportunities with diminishing states and expanding markets. World Development, v.21, n.4, p.607-622, 1993.

VIEIRA, D. L. M. et al. Agro-Sucessional restoration as a strategy to facilitate tropical forest recovery. Restoration Ecology, v.17, n.4, p.451-454, 2009. 